\title{
Wer bestimmt die Zukunft unseres Gesundheitswesens?
}

D. Güntert

Die letzten Monate waren für die Toggenburger Bevölkerung rund um das Spital Wattwil aufreibend und belastend. Die ganzen Diskussionen und Veranstaltungen mit verschiedensten Vertretern von Gemeinde und Kanton haben die Verunsicherung um die Zukunft des Spitals und der regionalen Gesundheitsversorgung mehr geschürt als beruhigt. Der kürzlich bekannt gewordene Verwaltungsratsbeschluss, der eine Schliessung zweier Regionalspitäler, namentlich Wattwil und Flawil, vorschlägt, hatte bei der Bevölkerung beider Regionen spontane und engagierte Reaktionen zur Folge. Mittels Demonstrationen in Flawil und Wattwil brachten die Bürger und Bürgerinnen ihren Widerstand gegen diesen Beschluss zum Ausdruck. Folge davon war der unverzügliche Rücktritt von Herrn Regierungsrat Anton Grüninger und die Einreichung einer Motion, die einen sofortigen Halt der Aktivität des bestehenden Verwaltungsrates fordert. Die neugewählte Regierungsrätin, Heidi Hanselmann, ist nun beauftragt, sich mit der aktuellen Problematik auseinanderzusetzen. Letztendlich liegt die Beschlusskompetenz für die Zukunft beider betroffener Spitäler bei der Regierung.

\section{Sparauftrag einzuhalten}

Nun stellt sich aber die Frage, aufgrund welcher Kriterien und Informationen kann, darf und muss die Regierung ihren Beschluss stützen. Die Regierung gibt vor, dass sie einen klaren Sparauftrag einhalten muss, der besagt, dass die Durchschnittskosten eines Patienten jährlich noch Fr. 6000.- betragen dürfen. Die bisherigen Durchschnittskosten der Region FürstenlandToggenburg lagen im Jahr 2003 bei Fr. 6894.-.

Die gebetsmühlenartig wiederholte Argumentation, dass wir uns unser «luxuriöses» Gesundheitssystem nicht mehr leisten können, weil die Schere zwischen Finanzierungsmöglichkeiten und medizinischen Leistungen stetig auseinanderklaffe und schuld daran hauptsächlich die High-Tech-Medizin sei, ist in aller Köpfe und nicht mehr zu revidieren. Werden Behauptungen wahrer, wenn sie einfach oft genug wiederholt werden?
Tatsache ist, dass die Gesundheitskosten seit dem 2. Weltkrieg stetig linear angestiegen sind, aber keineswegs exponentiell. Weitere Tatsache ist, dass die medizinische Entwicklung seit dem 2. Weltkrieg eine stetige Verbesserung in Diagnostik und Behandlung diverser Krankheiten zur Folge hatte und der heutige Stand der medizinischen Qualität direktes Resultat aus dieser Entwicklung ist. Der erwähnte Anstieg der Gesundheitskosten korreliert mit demjenigen anderer Bereiche, wie Lebenserhaltungskosten, usw.

\section{KVG-Revision und Krankenkassenprämien}

Mit der KVG-Revision hat die Erosion im Gesundheitswesen begonnen. Obwohl gegenteilig behauptet worden ist, sind die Krankenkassenprämien unverhältnismässig zu den erwähnten Gesundheitskosten angestiegen, nämlich von 1996 bis 2001 um über 30\%, die Kosten im Gesundheitswesen in der gleichen Periode um 21\% (Bundesamt für Statistik, Ausgabe 2003). Gut zwei Drittel der Gesundheitskosten von 46 Milliarden Franken werden von privaten Haushalten, d.h. vom Bürger, beglichen; der Rest wird von öffentlicher Hand, von Sozialversicherung, Sozialhilfe, Ergänzungsleistungen u.a. bezahlt. Mit dem KVG haben sich Bund und Kanton aus der Finanzierung der Gesundheitskosten herausgewunden. Also müssen diese Subventionen ausgeglichen werden. Diese Belastungen werden nun an die Leistungserbringer (Ärzte und Spitäler) nach unten abgewälzt. Die Frage stellt sich dabei, wohin denn nun die eingesparten Geldmengen fliessen?

\section{Praktische Auswirkungen des KVG}

Als direkt involvierter Arzt beobachte ich auf der praktischen Seite im alltäglichen Berufsleben, dass

- der administrative Aufwand verschiedenster Abläufe exponentiell zunimmt;

- Qualitätskontrollen, Leistungskontrollen, Zertifizierungen usw. exponentiell zunehmen; 
- Kontrollgremien, Consultingfirmen, Managementberater exponentiell zunehmen;

- Krankenkassenprämien exponentiell zunehmen;

- sinnvolle Krankenkassenleistungen stetig abnehmen;

- Leistungen wie Fitnesstraining, Lifestyleberatung, Reproduktionsmedizin, plastische Chirurgie u.a. in den Leistungskatalog von öffentlichen Spitälern neu aufgenommen werden;

- Alternativmedizin mit Evidence-based Medicine faktisch gleichgewertet wird;

- die Einführung eines neuen Tarifsystems (TARMED), deren Entwicklung etwa 15 Jahre dauerte und welches weder validiert noch in einem Pilotprojekt auf Tauglichkeit überprüft worden ist, aber auf Kosten der Ärzte vom Bund auferzwungen wurde;

- der Datenschutz für Patienten immer mehr unterhöhlt und teils auf krasse Weise missachtet wird;

- ärztliche Leistungen bis ins kleinste Detail elektronisch dokumentiert werden und deren Daten an Dritte oder Vierte oder ... weitergegeben werden müssen;

- Betriebswirtschafter zunehmend die Zukunft unserer Gesundheit dominieren;

- all diese Massnahmen bisher alles andere als einen Spareffekt zur Folge hatten und

- das Endresultat eine mittlerweile allgemein akzeptierte Zweiklassenmedizin ist, die aber erst am Anfang steht - der Anfang vom Ende?

\section{GD Zürich als Vorreiter der «Gesundheitsreform»}

Regierungsrätin Diener, namentlich Gesundheitsdirektorin des Kantons Zürich, gab erst kürzlich in einer Pressemitteilung den «neuen Tarif» durch, mit welchen Rationierungen der zukünftige Patient zu rechnen hat. Neben Stellenabbau und höherer Arbeitsbelastung für das ganze Personal ist eine «Senkung des Qualitätsstandards» unumgänglich. Es sei daran erinnert, dass dieselbe Regierungsrätin noch vor wenigen Jahren vehement eine solche Aussicht in Abrede gestellt hat. Was bedeutet aber Senkung des Qualitätsstandards konkret? Es bedeutet sicherlich nicht, dass die sogenannte und immer wieder beschuldigte High-Tech-Medizin betroffen ist. Denn diese High-Tech-Medizin betrifft einerseits nur einen sehr kleinen Teil der Patienten und fällt andererseits in den Gesamtgesundheitskosten gar nicht ins Gewicht. Was aber ins Gewicht fällt, ist der alte, mehrfach kranke Patient und der chronisch kranke Patient. Bei dieser grundversicherten Patientengruppe soll nun drastisch gespart werden. Dies bedeutet konkret, dass

- billigere und qualitativ schlechtere Medikamente verabreicht werden;

- nach bestimmten Kriterien, die noch offen sind, gewisse Leistungen verwehrt werden;

- Sterbebegleitung und palliative Medizin mehr in den Vordergrund gerückt werden;

- Hilfe zur Sterbehilfe toleriert wird;

- die freie Arztwahl voraussichtlich bald nicht mehr besteht;

- die Pflegequalität bei chronisch Kranken abnimmt;

- das Angebot an alternativen Behandlungen, die billiger im Vergleich zur Schulmedizin sind, ausgebaut wird.

\section{Zentralisierung als Zauberwort}

Etwas besser kommt der zusatzversicherte Patient weg. Ihm stehen gewisse Leistungen wie plastische Chirurgie, Lifestyleberatung, Alternativmedizin u.a.m. auf Aufpreis zur Verfügung. Entsprechend wollen sich nun die öffentlichen Spitäler, ganz in Anlehnung an die Privatkliniken, mehr an den Zusatzversicherten ausrichten, wohlgemerkt auf Kosten des Grundversicherten. Dies ist mitunter die Strategie der Verwaltungsräte der Spitalregionen im Kanton St. Gallen. Weiterhin soll nach internationalen Richtlinien eine drastische Zentralisierung stattfinden und meint damit nur noch wenige grosse und grössere Gesundheitszentren an Stelle der kleineren Regionalspitäler. Laut Argumentation der Betriebswirtschafter sind durch Zentralisierungen Doppelspurigkeiten in diversen Abläufen vermeidbar und könne dadurch gespart werden. Dies, obwohl unzählige anderweitige Erfahrungen das Gegenteil zeigen. In zentralen Grosskliniken findet in der Grundversorgung eine dramatische Mengenausweitung an medizinischen Leistungen statt, die in kleineren Regionalspitälern vermieden werden. Ganz zu schweigen, dass die Qualität der Pflege auf menschlicher Ebene eine ganz andere ist.

\section{Krankenkassen im Kampf gegen Ärzte}

Es ist ein offenes Geheimnis, dass die Krankenkassen die Ärzte als Sündenbock hinstellen und für die Misere im Gesundheitswesen verantwortlich machen. Sie lassen keine Strategie aus, um an mehr und detailliertere Patientendaten zu ge- 
langen. Damit sollen zuverlässigere Statistiken aufgestellt werden, um schliesslich verbindliche und zwingende Fallkostenpauschalen bestimmen zu können. Die Aktivität der Ärzte soll kontinuierlich eingeschränkt und überwacht werden. Die Aufhebung des Kontrahierungszwanges ist ein Mittel zu diesem Zweck. Bundesrat und Versicherer wollen auf jeden Fall die Aufhebung des Kontrahierungszwangs.

\section{Aufklärungsbedarf}

Die Entwicklung bzw. der stete Abbau der letzten Jahre in unserem Gesundheitswesen erfüllt mich mit grosser Sorge. Ich bin als Mediziner absolut der Ansicht, dass die Versorgung unserer Gesundheit nicht dem freien Markt übergeben wer- den darf, wie es die Ökonomen beabsichtigen. Ich bin aber auch der Ansicht, dass hier eine unlautere Strategie im Gange ist, die nicht wirklich eine Verbesserung der Gesundheitsversorgung zum Ziel hat, sondern Gelder abzweigt, die in andere Kanäle fliessen. Es sind zweifelsohne Gelder vorhanden, die es ermöglichen würden, den derzeitigen Standard aufrechtzuerhalten und weiterhin zu gewährleisten. Weiterhin bin ich der Ansicht, dass von politischer Seite der Bürger Anrecht auf Aufklärung hat, was bisher nicht geschehen ist. Dem Bürger muss klar das beabsichtigte Ziel inklusive deren Auswirkungen auf den einzelnen verständlich und unzweideutig dargelegt werden. Dann soll und kann der Bürger selbst entscheiden, welches Gesundheitssystem er möchte und zu welchem Preis. 